\title{
The Efficacy of New Chinese Diabetes Risk Score in Screening Undiagnosed Type 2 Diabetes and Prediabetes: A Community- Based Cross-Sectional Study in Eastern China
}

\author{
Tao Mao ${ }^{1 D},{ }^{1}$ Jiayan Chen, ${ }^{2,3}$ Haijian Guo, ${ }^{4}$ Chen Qu, ${ }^{1}$ Chu He, ${ }^{1}$ Xuepeng Xu, ${ }^{1}$ \\ Guoping Yang, ${ }^{1}$ Shiqi Zhen, ${ }^{1}$ and Xiaoning Li ${ }^{1}{ }^{1}$ \\ ${ }^{1}$ Department of Health Education, Jiangsu Provincial Center for Disease Control and Prevention, Nanjing 210009, China \\ ${ }^{2}$ School of Public Health, Nanchang University, Nanchang 330006, China \\ ${ }^{3}$ Jiangxi Province Key Laboratory of Preventive Medicine, Nanchang 330006, China \\ ${ }^{4}$ Department of Integrated Services, Jiangsu Provincial Center for Disease Control and Prevention, Nanjing 210009, China
}

Correspondence should be addressed to Xiaoning Li; 395777673@qq.com

Received 15 March 2020; Accepted 8 April 2020; Published 30 April 2020

Academic Editor: Suat Simsek

Copyright $\odot 2020$ Tao Mao et al. This is an open access article distributed under the Creative Commons Attribution License, which permits unrestricted use, distribution, and reproduction in any medium, provided the original work is properly cited.

\begin{abstract}
The New Chinese Diabetes Risk Score (NCDRS) is one of the recommended tools for screening undiagnosed type 2 diabetes in China. However, its performance in detecting undiagnosed diabetes needs to be verified in different community populations. Also, it is unknown whether NCDRS can be used in detecting prediabetes. In the present study, we aimed to evaluate the performance of NCDRS in detecting undiagnosed diabetes and prediabetes among the community residents in eastern China. We applied NCDRS in 7675 community residents aged 18-65 years old in Jiangsu Province. The results showed that the participants with undiagnosed diabetes reported the highest NCDRS value, followed by those with prediabetes $(P<0.001)$. The best cut-off points of NCDRS for detecting undiagnosed diabetes and prediabetes were 27 (with a sensitivity of $78.0 \%$ and a specificity of $57.7 \%$ ) and 27 (with a sensitivity of $66.0 \%$ and a specificity of $62.9 \%$ ). The AUCs of NCDRS for identifying undiagnosed diabetes and prediabetes were 0.749 (95\% CI: $0.739 \sim 0.759$ ) and 0.694 (95\% CI: 0.683 0.705). These results demonstrate the excellent performance of NCDRS in screening undiagnosed diabetes in the community population in eastern China and further provide evidence for using NCDRS in detecting prediabetes.
\end{abstract}

\section{Introduction}

With an increasing incidence and economic burden $[1,2]$, type 2 diabetes mellitus (DM) has been a significant public health concern worldwide. The global prevalence (age standardized) of DM has risen to $8.5 \%$ in the adult population in 2014 [3] and has grown fast in low- and middle-income countries over the past decade. Unfortunately, China is one of the countries facing serious challenges, with a prevalence of DM of 10.4\% (World Health Organization, WHO 1999 criteria) in the population aged 18 and above in 2013 [4]. However, only $36.5 \%$ of the patients in China were aware of their diabetes condition [4]. There is an urgent need to apply valid measures for the screening of DM.
The other major issue is the detection of prediabetes (preDM), including impaired glucose tolerance (IGT) and impaired fasting glycemia (IFG). PreDM is a transition between normal glucose tolerance and diabetes, and it has been proved to be a critical high-risk factor of DM. The prevalence of preDM had reached 35.7\% (American Diabetic Association, ADA 2003 criteria) in China in 2013 [4], which means that there may be a large number of patients with DM in the next few decades. However, the same as above, most of the individuals did not realize that they were at high risk of developing DM [5].

The previous studies demonstrate the significant roles of lifestyle intervention and early treatment in the prevention of DM [6-11], which highlight the importance of detecting DM 
and preDM at an early stage. However, the oral glucose tolerance test (OGTT) is complicated to implement, time-consuming, costly, and difficult to accept by most residents. In that, researchers have developed several DM risk assessments through nonlaboratory indicators for the rapid screening of undiagnosed DM in different populations [12-21]. There were also several DM risk measures developed for the Chinese population [22-25]. However, these measures were developed based on local conditions, and most of them did not consider issues such as gender differences. To provide a unified DM risk measurement, researchers developed a new assessment in 2013, the New Chinese Diabetes Risk Score (NCDRS) [26]. NCDRS is a DM risk assessment that includes age (years), gender, waist circumference (WC: $\mathrm{cm}$ ), body mass index (BMI: $\mathrm{kg} / \mathrm{m}^{2}$ ), systolic blood pressure (SBP: $\mathrm{mmHg}$ ), and family history of DM [26]. It has been recommended as a unified tool for screening of undiagnosed DM in China [27]. However, two questions remain about the performance of NCDRS. First, only a few studies have reported on the efficacy of NCDRS in the detection of DM. Of these studies, most have suffered from small sample sizes and some were even conducted in a single clinic; others focused only on older adults $[28,29]$. As we know, China is a multiethnic country with different economic levels and living habits. Research on the applicability of NCDRS in screening DM in different regions of China would be necessary. Second, to the best of our knowledge, no studies have evaluated the performance of NCDRS in screening preDM in a large sample of community residents in China. The efficacy of NCDRS in the detection of preDM requires further research.

Our study is aimed at filling the gap by evaluating the efficacy of NCDRS in the detection of undiagnosed DM and preDM among community residents in eastern China. In the present study, we conducted a community-based crosssectional investigation in Jiangsu Province, eastern China. Urban and rural residents aged between 18 and 65 years old were investigated. This study will enhance our understanding of the performance of NCDRS in screening undiagnosed DM in the Chinese population and shed new light on its efficacy in detecting preDM.

\section{Materials and Methods}

2.1. Study Design and Population. A community-based crosssectional study was conducted in Jiangsu Province of China from March to June 2016. The study population and procedures have been reported previously [30]. In brief, a fourstage stratified sampling method was used to select a representative sample of the general population in Jiangsu. A total of 8119 urban and rural residents aged between 18 and 65 years old were randomly selected from six districts or counties for the questionnaire survey, anthropometric measurement, and 2-hour oral glucose tolerance test $(2 \mathrm{~h}$ OGTT). People who have been diagnosed with diabetes, pregnancy, and severe mental disorders were excluded. Residents who failed to complete the questionnaire survey, anthropometric measurement, or blood specimen collection
TABLE 1: The scores of NCDRS.

\begin{tabular}{cccc}
\hline Indicators & Score & Indicators & Score \\
\hline Age (years) & & Waist circumference $(\mathrm{cm})$ \\
$20-24$ & 0 & $<75$ (men) or $<70$ (women) & 0 \\
$25-34$ & 4 & $75-79.9$ (men) or 70-74.9 (women) & 3 \\
$35-39$ & 8 & $80-84.9$ (men) or 75-79.9 (women) & 5 \\
$40-44$ & 11 & $85-89.9$ (men) or $80-84.9$ (women) & 7 \\
$45-49$ & 12 & $90-94.9$ (men) or 85-89.9 (women) & 8 \\
$50-54$ & 13 & $\geq 95$ (men) or $\geq 90$ (women) & 10 \\
$55-59$ & 15 & Systolic blood pressure (mmHg) & \\
$60-64$ & 16 & $<110$ & 0 \\
$65-84$ & 18 & $110-119$ & 1 \\
BMI (kg/m ${ }^{2}$ ) & & $120-129$ & 3 \\
$<22$ & 0 & $130-139$ & 6 \\
$22-23.9$ & 1 & $140-149$ & 7 \\
$24-29.9$ & 3 & $150-159$ & 8 \\
$\geq 30$ & 5 & $\geq 160$ & 10 \\
Sex & & Family history of diabetes, yes vs. no & \\
Women & 0 & No & 6 \\
Men & 2 & Yes & 6 \\
\hline
\end{tabular}

Note: "articipants aged 18 and 19 scored 0 for "Age" in the present study.

were excluded from data analysis. Finally, a total of 7675 residents were included in the analysis.

2.2. New Chinese Diabetes Risk Score (NCDRS). The scores of NCDRS are shown in Table 1 . The total score ranged from 0 to 51 , and the recommended optimal cut-off point for undiagnosed DM was 25 [26].

2.3. Data Collection. The study was conducted in local township health centers by trained research staff. A standard questionnaire was used, including personal information such as sociodemographic characteristics and family history of DM. Anthropometric measurements were taken by trained observers, included body weight, height, waist circumference, and blood pressure.

2.4. Laboratory Measurements. A $5 \mathrm{~mL}$ blood specimen was obtained for fasting plasma glucose (FPG) measurement after at least 8 hours of fasting. The other $5 \mathrm{~mL}$ blood specimen was obtained for 2-hour plasma glucose (2 h PG) measurement after a $75 \mathrm{~g}$ anhydrous glucose load for two hours. As the six districts or counties were geographically distant, the laboratory tests of plasma glucose (PG) were carried out by local people's hospitals at the county or the center for disease control and prevention. The instruments and methods used to measure PG have been reported previously [30].

2.5. DM and PreDM Definition. According to the 1999 World Health Organization (WHO) criteria, DM was defined as $\mathrm{FPG} \geq 7.0 \mathrm{mmol} / \mathrm{L}$ or $2 \mathrm{hPG} \geq 11.1 \mathrm{mmol} / \mathrm{L}$. PreDM was defined as $6.1 \mathrm{mmol} / \mathrm{L} \leq \mathrm{FPG}<7.0 \mathrm{mmol} / \mathrm{L}$ or $7.8 \mathrm{mmol} / \mathrm{L}$ $\leq 2 \mathrm{hPG}<11.1 \mathrm{mmol} / \mathrm{L}$. Normal glucose tolerance was defined as FPG $<6.1 \mathrm{mmol} / \mathrm{L}$ and $2 \mathrm{~h} \mathrm{PG}<7.8 \mathrm{mmol} / \mathrm{L}$. 
2.6. Ethical Consideration. This study was approved by the Ethics Review Committee of Jiangsu Provincial Center for Disease Control and Prevention (No. JSJK2016-B003-03). Informed consent was obtained from each participant.

2.7. Statistical Analysis. Continuous and categorical variables were described by mean \pm standard deviation $(X \pm S D)$ and percentage (\%). According to different glucose metabolism levels, the characteristics of each group were described. Comparisons among the groups were made with one-way ANOVA for continuous variables and Chi-square test for proportions. Sensitivity, specificity, Youden index, positive predictive value, and negative predictive value were calculated for each NCDRS value from 25 to 32 for screening undiagnosed DM, preDM, or both of them. The optimal cut-off point was determined by the highest Youden index. The receiver operating characteristic (ROC) curves by gender and age groups were used to present the performance of NCDRS in screening both undiagnosed DM and preDM. The area under the curve (AUC) was used to evaluate the screening effect of NCDRS. All statistical analyses were performed in SPSS 17.0 and MedCalc18.11.3.

\section{Results}

The characteristics of the participants were presented in Table 2. The average age of the participants was $43.8 \pm$ 11.9 years old, and $56.0 \%$ were female. Of the participants, $41.7 \%$ had a middle school education, and $39.0 \%$ were farmers. $16.8 \%$ of the participants reported that they had a family history of diabetes. Among all participants, the average BMI, WC, SBP, DBP, FPG, and $2 \mathrm{~h}$ PG were $25.1 \pm$ $4.0 \mathrm{~kg} / \mathrm{m}^{2}, 83.5 \pm 10.4 \mathrm{~cm}, 128.2 \pm 18.8 \mathrm{mmHg}, 79.2 \pm 11.7$ $\mathrm{mmHg}, 5.5 \pm 1.0 \mathrm{mmol} / \mathrm{L}$, and $6.6 \pm 2.5 \mathrm{mmol} / \mathrm{L}$, respectively. The average NCDRS score was $24.1 \pm 9.3$, and $52.1 \%$ of the participants reported a high-risk score (NCDRS $\geq 25$ ). The detection rates of undiagnosed DM and preDM were 6.5\% (95\% CI: $5.96 \%$ to $7.07 \%$ ) and $16.7 \%$ (95\% CI: $15.91 \%$ to $17.58 \%)$. The detection rates for $\mathrm{DM}$ and preDM in the high-risk group (NCDRS $\geq 25$ ) were $10.6 \%$ and $23.6 \%$, while the detection rates in the low-risk group (NCDRS $<25$ ) were $2.1 \%$ and $9.3 \%$. By using the LSD method, the NCDRS scores of DM were higher than the scores of preDM $(P<0.001)$, and the scores of preDM were higher than the scores of normal glucose tolerance residents $(P<0.001)$. There was a certain relationship between the NCDRS scores and the glucose metabolism levels.

As shown in Table 3, the optimal cut-off point for the detection of undiagnosed DM was 31 for men (sensitivity = $65.2 \%$, specificity $=69.0 \%$ ) and 27 for women (sensitivity = $76.2 \%$, specificity $=63.5 \%$ ). For preDM, the optimal cut-off point was 29 for men (sensitivity $=61.1 \%$, specificity $=$ $64.7 \%$ ) and 26 for women (sensitivity $=65.7 \%$, specificity $=$ $64.5 \%$ ). When screening both undiagnosed DM and preDM, the best cut-off point was 27 for men (sensitivity $=73.5 \%$, specificity $=55.6 \%$ ) and 26 for women (sensitivity $=69.3 \%$, specificity $=64.5 \%)$. In the total sample, the optimal cut-off point for screening undiagnosed DM was 27 (sensitivity $=$ $78.0 \%$, specificity $=57.7 \%$, Youden index $=0.357$ ), same as for the detection of preDM (sensitivity $=66.0 \%$, specificity $=$ $62.9 \%$, Youden index $=0.289$ ). At the cut-off point of $25 \mathrm{rec}$ ommended by NCDRS, sensitivity, specificity, and Youden index of detecting undiagnosed DM were $84.8 \%, 50.1 \%$, and 0.349 , respectively. The PPV of NCDRS was $11.4 \%$ for DM and $20.8 \%$ for preDM.

The areas under the ROC curves (AUCs) for identifying undiagnosed DM were 0.731 in men and 0.761 in women $(P=0.139)$. The AUC of the $\geq 45$ age group was smaller than that of the other age groups (all $P<0.05$ ) (see Figures 1(a) and $1(\mathrm{~b})$ ). The AUCs for identifying preDM were 0.683 in men and 0.698 in women $(P=0.325)$. There was a statistically significant difference in the AUCs between the 4554 age group and the $\leq 44$ age group (all $P<0.05$ ) (see Figures $1(\mathrm{c})$ and $1(\mathrm{~d})$ ). The AUCs for identifying undiagnosed DM and preDM were 0.749 (95\% CI: 0.739 0.759) and 0.694 (95\% CI: 0.683 0.705). The AUC for identifying both undiagnosed $\mathrm{DM}$ and preDM was 0.718 (95\% CI: $0.708 \sim 0.728$ ) in the total sample.

\section{Discussion}

One of the aims of our study was to evaluate the performance of NCDRS in screening undiagnosed DM among community residents in eastern China. As we expected, NCDRS showed excellent validity in detecting undiagnosed DM. The AUC for identifying undiagnosed DM was 0.749 in this study, which was similar to 0.748 reported in the China National Diabetes and Metabolic Disorders Study [26]. However, the optimal cut-off point for undiagnosed DM in this study was 27, two points higher than the recommended cut-off point of 25 in the national survey above. In previous studies [28, 29 ], the best cut-off point was 33 in the population aged over 60 years and 20 in the hospital physical examination group. The possible cause for these differences may be attributed to the differences in age, regions, and other demographic characteristics among these findings. It indicates that, as we mentioned above, studies on the applicability of NCDRS in screening DM in different regions and populations of China is necessary. We found that, when using for the screening of undiagnosed DM, the sensitivity and specificity of NCDRS at the optimal cut-off point of 27 in this study were $78.0 \%$ and $57.7 \%$; while at the recommended cut-off point of 25 , the sensitivity and specificity were $84.8 \%$ and $50.1 \%$. It demonstrates that, when using the cut-off value of 27 in community residents in eastern China, the sensitivity may decrease. Still, the specificity will improve, which could reduce the proportion of individuals who are misjudged as DM. Taken together, these findings suggest that a cut-off score of 27 is more applicable if we seek to maximize the effects of screening. However, given the severe consequences of missed diagnosis, a cut-off point of 25 , so as to improve the sensitivity of detection, is also recommended in practice.

Moreover, NCDRS had a low PPV (11.4\%) for DM in our study, which was similar to $11.8 \%$ reported in the national survey above [26]. As we know, more benefits of further diagnosis for individuals who were screening positive could be obtained as PPV increased. However, the PPV was closely related to the prevalence of the target disease in the tested 
TABLE 2: Characteristics of the participants included in the study, by glucose metabolism levels.

\begin{tabular}{|c|c|c|c|c|c|}
\hline & Overall $(n=7675)$ & Normal glucose tolerance $(n=5890)$ & $\operatorname{PreDM}(n=1285)$ & $\mathrm{DM}(n=500)$ & $P$ value \\
\hline \multicolumn{6}{|l|}{ Gender } \\
\hline Male & $3374(44.0)$ & $2486(42.2)$ & $632(49.2)$ & $256(51.2)$ & \multirow{2}{*}{$<0.001^{\mathrm{b}}$} \\
\hline Female & $4301(56.0)$ & $3404(57.8)$ & $653(50.8)$ & $244(48.8)$ & \\
\hline Age (years) & $43.8 \pm 11.9$ & $42.3 \pm 11.9$ & $48.3 \pm 10.6$ & $49.4 \pm 10.0$ & $<0.001^{\mathrm{a}}$ \\
\hline \multicolumn{6}{|l|}{ Age group } \\
\hline $18-24$ & $385(5.0)$ & $354(6.0)$ & $24(1.9)$ & $7(1.4)$ & \multirow{5}{*}{$<0.001^{\mathrm{b}}$} \\
\hline $25-34$ & $1620(21.1)$ & $1440(24.4)$ & $136(10.6)$ & $44(8.8)$ & \\
\hline $35-44$ & $1603(20.9)$ & $1284(21.8)$ & $238(18.5)$ & $81(16.2)$ & \\
\hline $45-54$ & $2552(33.3)$ & $1844(31.3)$ & $504(39.2)$ & $204(40.8)$ & \\
\hline $55-65$ & $1515(19.7)$ & $968(16.4)$ & $383(29.8)$ & $164(32.8)$ & \\
\hline \multicolumn{6}{|l|}{ Education level } \\
\hline Below primary school & $784(10.2)$ & $520(8.8)$ & $206(16.0)$ & $58(11.6)$ & \multirow{5}{*}{$<0.001^{\mathrm{b}}$} \\
\hline Primary school & $1332(17.4)$ & $951(16.1)$ & $280(21.8)$ & $101(20.2)$ & \\
\hline Middle school & $3203(41.7)$ & $2462(41.8)$ & $509(39.6)$ & $232(46.4)$ & \\
\hline High school & $1630(21.2)$ & $1323(22.5)$ & $218(17.0)$ & $89(17.8)$ & \\
\hline College and above & $726(9.5)$ & $634(10.8)$ & $72(5.6)$ & $20(4.0)$ & \\
\hline \multicolumn{6}{|l|}{ Occupation } \\
\hline Public institution officer ${ }^{c}$ & $1061(13.8)$ & $854(14.5)$ & $162(12.6)$ & $45(9.0)$ & \multirow{4}{*}{$<0.001^{\mathrm{b}}$} \\
\hline Company employee & $2231(29.1)$ & $1801(30.6)$ & $308(24.0)$ & $122(24.4)$ & \\
\hline Farmer & $2995(39.0)$ & $2093(35.5)$ & $642(50.0)$ & $260(52.0)$ & \\
\hline Other & $1388(18.1)$ & $1142(19.4)$ & $173(13.5)$ & $73(14.6)$ & \\
\hline \multicolumn{6}{|l|}{ Family history of diabetes } \\
\hline Yes & $1290(16.8)$ & $920(15.6)$ & $248(19.3)$ & $122(24.4)$ & \multirow{2}{*}{$<0.001^{\mathrm{b}}$} \\
\hline No & $6385(83.2)$ & $4970(84.4)$ & $1037(80.7)$ & $378(75.6)$ & \\
\hline BMI $\left(\mathrm{kg} / \mathrm{m}^{2}\right)$ & $25.1 \pm 4.0$ & $24.7 \pm 3.9$ & $26.3 \pm 3.6$ & $27.4 \pm 3.9$ & $<0.001^{\mathrm{a}}$ \\
\hline $\mathrm{WC}(\mathrm{cm})$ & $83.5 \pm 10.4$ & $82.2 \pm 10.3$ & $86.8 \pm 9.7$ & $90.3 \pm 10.0$ & $<0.001^{\mathrm{a}}$ \\
\hline SBP (mmHg) & $128.2 \pm 18.8$ & $125.7 \pm 18.0$ & $134.9 \pm 18.9$ & $140.4 \pm 18.1$ & $<0.001^{\mathrm{a}}$ \\
\hline DBP (mmHg) & $79.2 \pm 11.7$ & $77.8 \pm 11.4$ & $82.8 \pm 11.7$ & $85.2 \pm 10.9$ & $<0.001^{\mathrm{a}}$ \\
\hline FPG (mmol/L) & $5.5 \pm 1.0$ & $5.2 \pm 0.4$ & $5.9 \pm 0.6$ & $7.8 \pm 2.1$ & $<0.001^{\mathrm{a}}$ \\
\hline $2 \mathrm{~h} \mathrm{PG}(\mathrm{mmol} / \mathrm{L})$ & $6.6 \pm 2.5$ & $5.6 \pm 1.0$ & $8.2 \pm 1.5$ & $13.4 \pm 4.1$ & $<0.001^{\mathrm{a}}$ \\
\hline NCDRS value & $24.1 \pm 9.3$ & $22.5 \pm 9.2$ & $28.7 \pm 7.4$ & $31.5 \pm 6.7$ & $<0.001^{\mathrm{a}}$ \\
\hline \multicolumn{6}{|l|}{ NCDRS group } \\
\hline Low-risk (NCDRS < 25) & $3673(47.9)$ & $3255(55.3)$ & $342(26.6)$ & $76(15.2)$ & \multirow{2}{*}{$<0.001^{\mathrm{b}}$} \\
\hline High-risk (NCDRS $\geq 25$ ) & $4002(52.1)$ & $2635(44.7)$ & $943(73.4)$ & $424(84.8)$ & \\
\hline
\end{tabular}

Note: data are reported as mean \pm standard deviation or frequency (percent (\%)); BMI=body mass index; WC=waist circumference; $\mathrm{SBP}=$ systolic blood

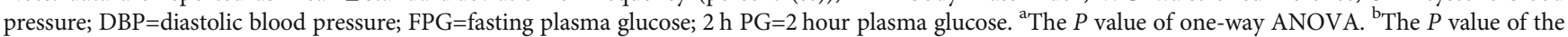
Chi-square test. ${ }^{c}$ Public institution officers include government officers, teachers, medical staffs, and other public institution officers.

population. When sensitivity and specificity were constant, PPV increased with the risen prevalence of the target disease. One previous study reported that the sensitivity and specificity of NCDRS were $70.5 \%$ and $53.0 \%$ in Chinese adults aged 45-70 with an undiagnosed DM prevalence of $15.3 \%$ [25], resulting in a PPV of $21.3 \%$. It indicates that using NCDRS in a population with a higher prevalence of undiagnosed DM will result in higher benefits. Therefore, it is better to use NCDRS for screening the high-risk group of DM in the future.

To the best of our knowledge, this study is the first to evaluate the efficacy of NCDRS in screening preDM in a large sample of representative community residents in China. We found that the AUC of NCDRS in screening preDM was 0.694, indicating that NCDRS also performs well in the detection of preDM. Compared with the cut-off points for the detection of undiagnosed DM, the optimal cut-off scores for the detection of preDM were two points lower in men and one point lower in women. However, in the total sample, the optimal cut-off point for screening preDM was 27, which shared the same value with screening undiagnosed DM. It is an interesting finding that NCDRS may differ from other DM risk assessments. For example, in both the ADA scores and CDC scores, the cut-off points for preDM were one point 


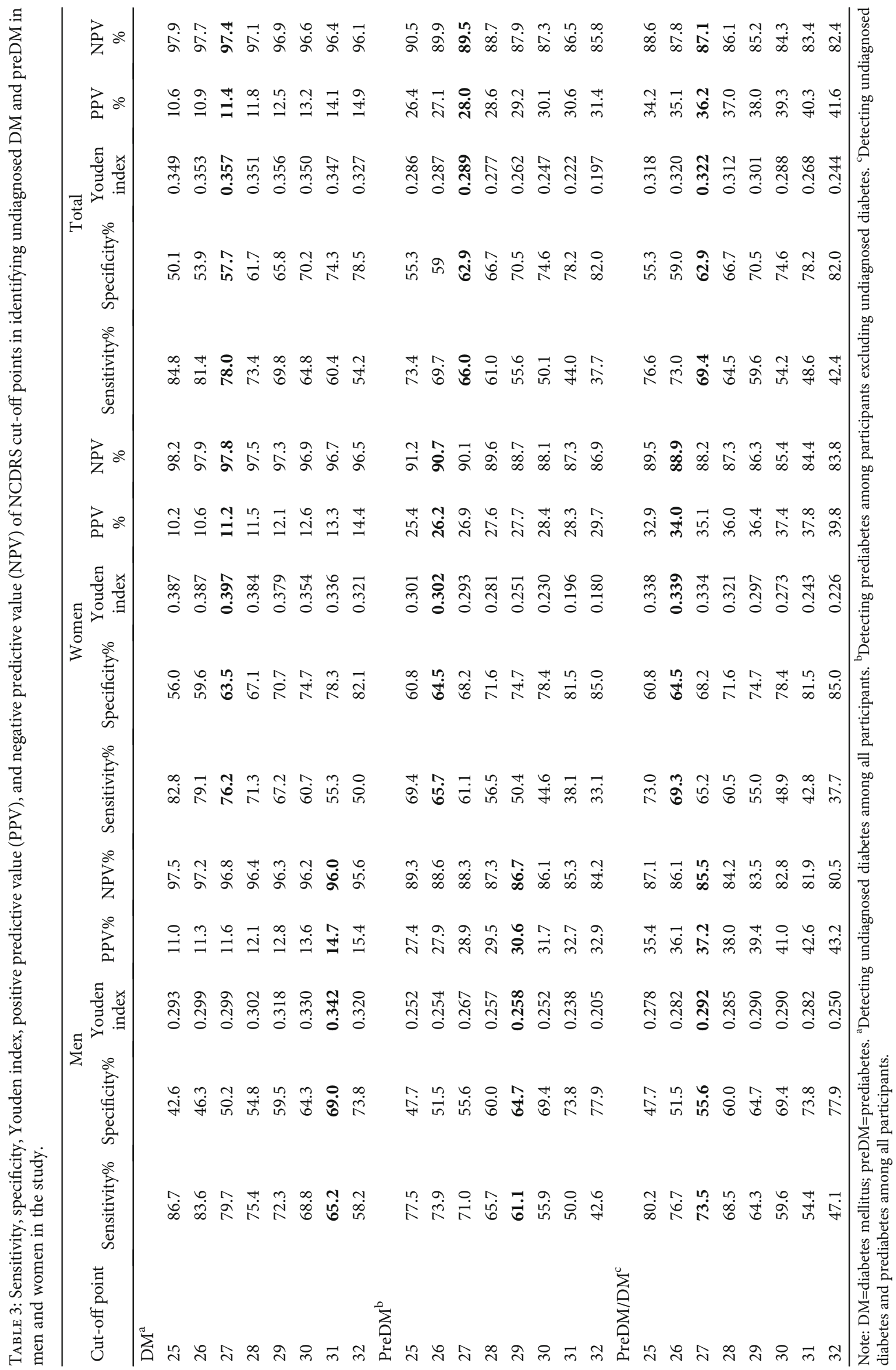



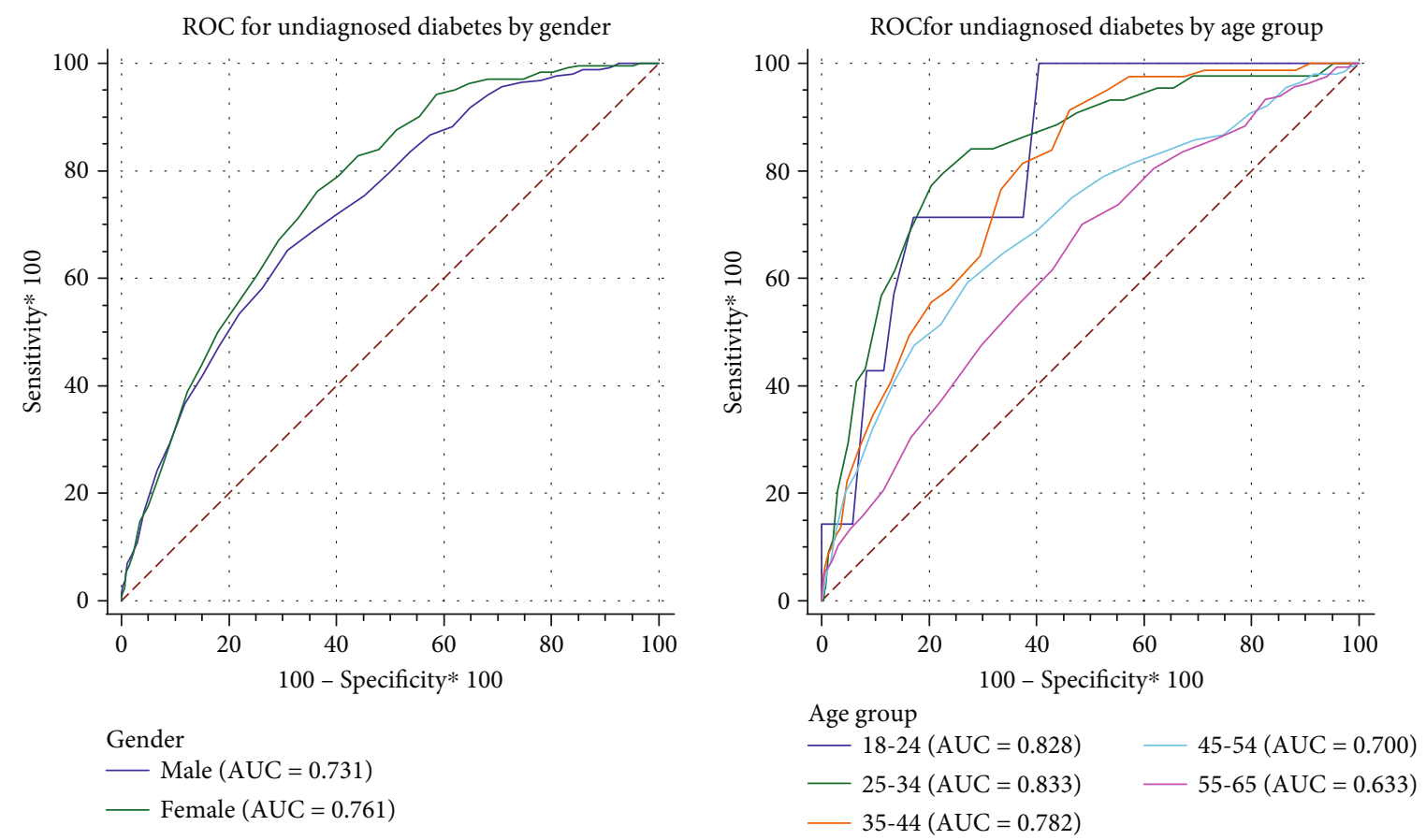

(a)

(b)
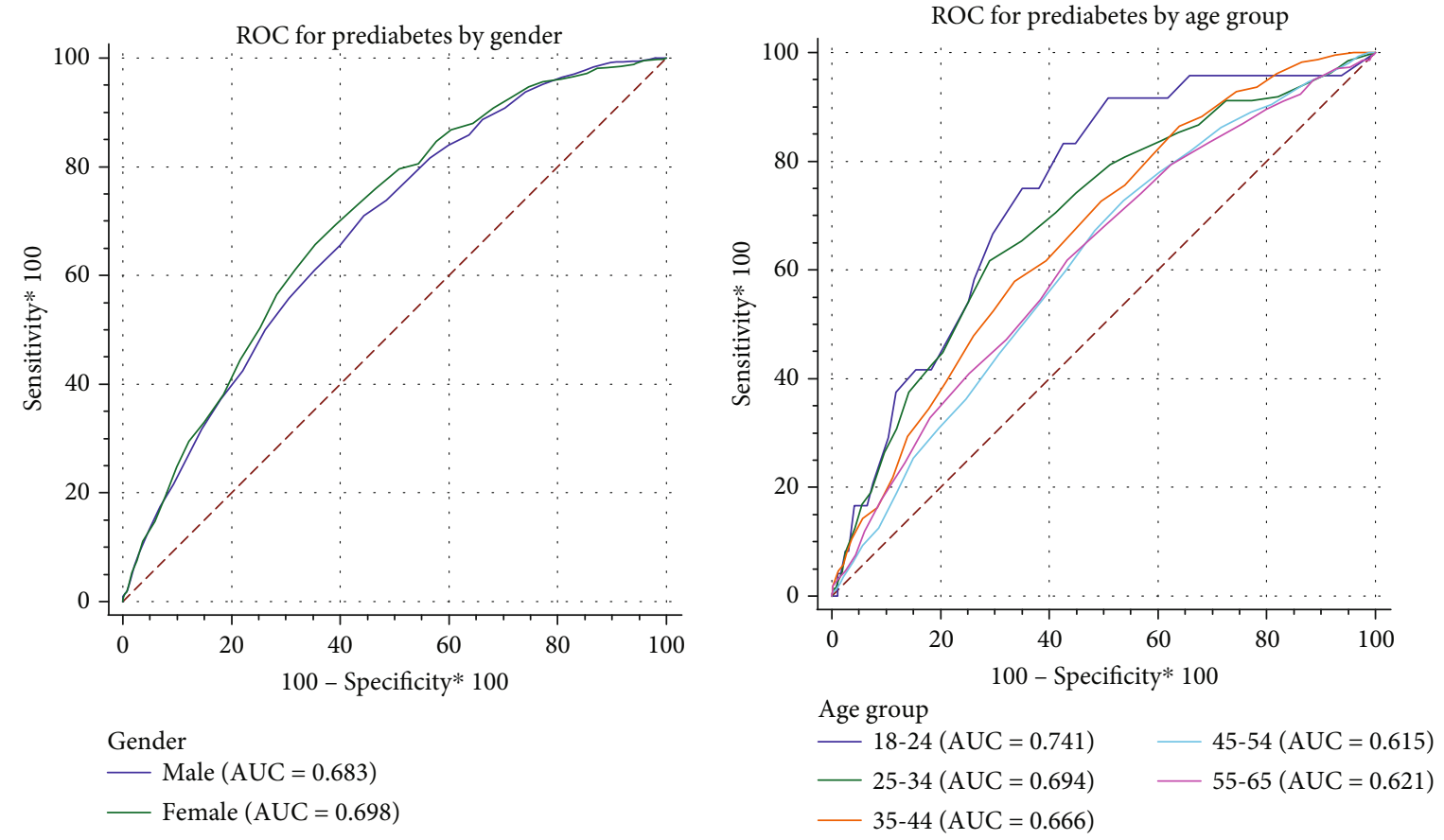

(c)

(d)

Figure 1: Receiver operating characteristic (ROC) curve for identifying undiagnosed diabetes ( $a$ and b) and prediabetes (c and d) by gender and age group in the study.

lower than the points for DM [31]. It may be due to the fact that preDM shares common risk factors with DM in eastern China [32, 33]. Nevertheless, using the same cut-off point for the detection of DM and preDM makes NCDRS more convenient and applicable for practice.

There was no statistically significant difference in the AUC of NCDRS in detecting undiagnosed DM and preDM between men and women. However, the AUC showed statistically significant differences in identifying undiagnosed DM and preDM among different age groups. The performance of NCDRS was more effective in the $\leq 44$ age group compared with the $\geq 45$ age group in both DM and preDM. The previous study has shown that the DM risk assessments could neither provide efficient screening of DM, nor could they 
identify individuals with elevated blood glucose levels in an older population ( $\geq 55$ years old) [34]. That is, like other assessments, NCDRS may be more applicable in a younger population. It means that NCDRS could play a significant role in early detection, early diagnosis, and early treatment, in which a higher return would be expected.

Several limitations to the current study need to be acknowledged. First, the tests of plasma glucose were conducted in different laboratories, which may result in slight differences in the value of plasma glucose. However, all of the tests were carried out by the local public hospital or the CDC. All laboratories of these institutes meet the requirements of the Administrative Measures for Clinical Laboratory of Medical Institutions. The instruments and reagents were also calibrated. Second, although we have taken efforts to collect samples from both urban and rural areas, the samples were only from one province in eastern China, which raises concerns about the possible selection bias. Further research in different regions is needed. Third, due to the nature of the cross-sectional study, we were unable to evaluate the performance of NCDRS in predicting DM and preDM events. We will try to conduct a follow-up study and assess the predictive validity of NCDRS further.

\section{Conclusions}

In conclusion, this study is the first to assess the efficacy of NCDRS in the screening of undiagnosed DM and preDM in a large sample of community residents in eastern China. The findings suggest that NCDRS performs well in screening both undiagnosed DM and preDM. Our study not only supports the excellent efficacy of NCDRS in the detection of undiagnosed DM but also highlights its potential usefulness in detecting preDM.

\section{Data Availability}

The data used to support the findings of this study are available from the corresponding author upon request.

\section{Conflicts of Interest}

The authors declare that there is no potential conflict of interest regarding the publication of this paper.

\section{Authors' Contributions}

Tao Mao and Jiayan Chen contributed equally to this work.

\section{Acknowledgments}

The authors gratefully acknowledge the assistance from the medical staff at local township health centers in Jiangsu Province and the support from the community health management teams who participated in the project. This study was funded by the World Diabetes Foundation (grant number 15-967).

\section{References}

[1] International Diabetes Federation, IDF Diabetes Atlas, International Diabetes Federation, Brussels, Belgium, 7th edition, 2015.

[2] E. Q. Wu, J. Xie, E. X. Du, M. Liu, and G. Liu, "Trend in economic burden of diabetes in urban China from 2009 to 2011," Value in Health, vol. 16, no. 3, p. A161, 2013.

[3] WHO, Global report on diabetes: 1. Diabetes mellitus-epidemiology, World Health Organization, Geneva, Switzerland, 2016.

[4] L. Wang, P. Gao, M. Zhang et al., "Prevalence and ethnic pattern of diabetes and prediabetes in China in 2013," JAMA, vol. 317, no. 24, pp. 2515-2523, 2017.

[5] Q. Zhuang, L. Wu, Y. Lu, J. Du, and G. Guo, "Awareness and intervention status of prediabetes among Chinese adults: implications from a community-based investigation," International Journal of Clinical and Experimental Medicine, vol. 8, no. 3, pp. 4480-4486, 2015.

[6] X. R. Pan, G. W. Li, Y. H. Hu et al., "Effects of diet and exercise in preventing NIDDM in people with impaired glucose tolerance-the Da Qing IGT and Diabetes Study," Diabetes Care, vol. 20, no. 4, pp. 537-544, 1997.

[7] J. Tuomilehto, J. Lindström, J. G. Eriksson et al., "Prevention of type 2 diabetes mellitus by changes in lifestyle among subjects with impaired glucose tolerance," New England Journal of Medicine, vol. 344, no. 18, pp. 1343-1350, 2001.

[8] K. Kosaka, M. Noda, and T. Kuzuya, "Prevention of type 2 diabetes by lifestyle intervention: a Japanese trial in IGT males," Diabetes Research and Clinical Practice, vol. 67, no. 2, pp. 152-162, 2005.

[9] T. Saaristo, L. Moilanen, E. Korpi-Hyovalti et al., "Lifestyle intervention for prevention of type 2 diabetes in primary health care: one-year follow-up of the Finnish National Diabetes Prevention Program (FIN-D2D)," Diabetes Care, vol. 33, no. 10, pp. 2146-2151, 2010.

[10] D. König, J. Hörmann, H. Predel, and A. Berg, “A 12-month lifestyle intervention program improves body composition and reduces the prevalence of prediabetes in obese patients," Obesity Facts, vol. 11, no. 5, pp. 393-399, 2018.

[11] W. H. Herman, W. Ye, S. J. Griffin et al., "Early detection and treatment of type 2 diabetes reduce cardiovascular morbidity and mortality: a simulation of the results of the Anglo-Danish-Dutch Study of Intensive Treatment in People with Screen-Detected Diabetes in Primary Care (ADDITION-Europe)," Diabetes Care, vol. 38, no. 8, pp. 14491455, 2015.

[12] T. Saaristo, M. Peltonen, J. Lindström et al., "Cross-sectional evaluation of the Finnish Diabetes Risk Score: a tool to identify undetected type 2 diabetes, abnormal glucose tolerance and metabolic syndrome," Diabetes \& Vascular Disease Research, vol. 2, no. 2, pp. 67-72, 2005.

[13] W. Aekplakorn, P. Bunnag, M. Woodward et al., "A risk score for predicting incident diabetes in the Thai population," Diabetes Care, vol. 29, no. 8, pp. 1872-1877, 2006.

[14] Y. Doi, T. Ninomiya, J. Hata et al., "Two risk score models for predicting incident type 2 diabetes in Japan," Diabetic Medicine, vol. 29, no. 1, pp. 107-114, 2012.

[15] M. Riaz, A. Basit, M. Z. I. Hydrie et al., "Risk assessment of Pakistani individuals for diabetes (RAPID)," Primary Care Diabetes, vol. 6, no. 4, pp. 297-302, 2012. 
[16] C. P. Rowan, L. A. Miadovnik, M. C. Riddell, M. A. Rotondi, N. Gledhill, and V. K. Jamnik, "Identifying persons at risk for developing type 2 diabetes in a concentrated population of high risk ethnicities in Canada using a risk assessment questionnaire and point-of-care capillary blood $\mathrm{HbA}_{1 \mathrm{c}}$ measurement," BMC Public Health, vol. 14, no. 1, 2014.

[17] P. L. Hu, Y. L. E. Koh, and N. C. Tan, "The utility of diabetes risk score items as predictors of incident type 2 diabetes in Asian populations: an evidence-based review," Diabetes Research and Clinical Practice, vol. 122, pp. 179-189, 2016.

[18] F. Yan, E. Cha, E. T. Lee, R. M. Mayberry, W. Wang, and G. Umpierrez, "A self-assessment tool for screening young adults at risk of type 2 diabetes using Strong Heart Family Study data," The Diabetes Educator, vol. 42, no. 5, pp. 607-617, 2016.

[19] R. S. Patil and J. S. Gothankar, "Assessment of risk of type 2 diabetes using the Indian Diabetes Risk Score in an urban slum of Pune, Maharashtra, India: a cross-sectional study," WHO South-East Asia Journal of Public Health, vol. 5, no. 1, pp. 53-61, 2016.

[20] G. J. Félix-Martínez and R. Godínez-Fernández, "Screening models for undiagnosed diabetes in Mexican adults using clinical and self-reported information," Endocrinología y Nutrición, vol. 65, no. 10, pp. 603-610, 2018.

[21] R. Rajput, K. Garg, and M. Rajput, "Prediabetes Risk Evaluation Scoring System (PRESS): a simplified scoring system for detecting undiagnosed prediabetes," Primary Care Diabetes, vol. 13, no. 1, pp. 11-15, 2019.

[22] W. G. Gao, Y. H. Dong, Z. C. Pang et al., "A simple Chinese risk score for undiagnosed diabetes," Diabetic Medicine, vol. 27, no. 3, pp. 274-281, 2010.

[23] J. Xie, D. Hu, D. Yu, C. S. Chen, J. He, and D. Gu, "A quick selfassessment tool to identify individuals at high risk of type 2 diabetes in the Chinese general population," Journal of Epidemiology \& Community Health, vol. 64, no. 3, pp. 236-242, 2010.

[24] S. Luo, L. Han, P. Zeng et al., "A risk assessment model for type 2 diabetes in Chinese," PLoS One, vol. 9, no. 8, article e104046, 2014.

[25] J. Wu, X. Hou, L. Chen et al., "Development and validation of a non-invasive assessment tool for screening prevalent undiagnosed diabetes in middle-aged and elderly Chinese," Preventive Medicine, vol. 119, pp. 145-152, 2019.

[26] X. Zhou, Q. Qiao, L. Ji et al., "Nonlaboratory-based risk assessment algorithm for undiagnosed type 2 diabetes developed on a nation-wide diabetes survey," Diabetes Care, vol. 36, no. 12, pp. 3944-3952, 2013.

[27] Chinese Diabetes Society, "National guidelines for the prevention and control of type 2 diabetes (2013)," Chinese Journal of Diabetes Mellitus, vol. 6, no. 7, pp. 447-498, 2014.

[28] L. Li, S. Hu, and H. Hong, "Evaluation on screening effects of diabetes mellitus and impaired glucose regulation for two diabetes screening questionnaires," Chinese Journal of Prevention and Control of Chronic Diseases, vol. 23, no. 4, pp. 256-258, 2015.

[29] L. Wang, J. Wang, A. Liu et al., "Evaluating the applied effectiveness of Chinese diabetes risk score in physical examination of elderly population," Chinese Journal of Geriatrics, vol. 36, no. 7, pp. 806-810, 2017.

[30] J. Chen, H. Guo, S. Yuan et al., "Efficacy of urinary glucose for diabetes screening: a reconsideration," Acta Diabetologica, vol. 56, no. 1, pp. 45-53, 2019.
[31] E. Poltavskiy, D. J. Kim, and H. Bang, "Comparison of screening scores for diabetes and prediabetes," Diabetes Research and Clinical Practice, vol. 118, pp. 146-153, 2016.

[32] M. Zhao, H. Lin, Y. Yuan et al., "Prevalence of pre-diabetes and its associated risk factors in rural areas of Ningbo, China," International Journal of Environmental Research and Public Health, vol. 13, no. 8, p. 808, 2016.

[33] Y. Song, X. Zhang, H. Zhang et al., "Prevalence of diabetes and prediabetes in adults from a third-tier city in eastern China: a cross-sectional study," Diabetes Therapy, vol. 10, no. 4, pp. 1473-1485, 2019.

[34] T. Featherstone, D. T. Eurich, and S. H. Simpson, "Limited effectiveness of diabetes risk assessment tools in seniors' facility residents," Value in Health, vol. 20, no. 3, pp. 329-335, 2017. 